\title{
Cholinergic partition cells and lamina $X$ neurons induce a muscarinic-dependent short-term potentiation of commissural glutamatergic inputs in lumbar motoneurons
}

\section{Sandrine S. Bertrand * and Jean-René Cazalets}

CNRS UMR 5287, INCIA, Université de Bordeaux, Bordeaux, France

\author{
Edited by: \\ Abdel El Manira, Karolinska Institute, \\ Sweden \\ Reviewed by: \\ Gareth Miles, University of \\ St Andrews, UK \\ Réjean Dubuc, Université du Québec \\ à Montréal, Canada \\ *Correspondence: \\ Sandrine S. Bertrand, Centre National \\ de la Recherche Scientifique, Institut \\ de Neurosciences Cognitives et \\ Intégratives d'Aquitaine, Université \\ Victor Ségalen (Bordeaux 2), Zone \\ Nord, Bât. 2A/B, 146, rue Léo Saignat, \\ 33076 Bordeaux cedex, France. \\ e-mail: sandrine.bertrand@ \\ u-bordeaux2.fr
}

Acetylcholine and the activation of muscarinic receptors influence the activity of neural networks generating locomotor behavior in the mammalian spinal cord. Using electrical stimulations of the ventral commissure, we show that commissural muscarinic (CM) depolarizations could be induced in lumbar motoneurons. We provide a detailed electrophysiological characterization of the muscarinic receptors and the membrane conductance involved in these responses. Activation of the $\mathrm{CM}$ terminals, originating from lamina $X$ neurons and partition cells, induced a pathway-specific short-term potentiation (STP) of commissural glutamatergic inputs in motoneurons. This STP is occluded in the presence of the muscarinic antagonist atropine. During fictive locomotion, the activation of the commissural pathways transiently enhanced the motor output in a muscarinic-dependent manner. This study describes for the first time a novel regulatory mechanism of synaptic strength in spinal locomotor networks. Such cellular mechanisms would endow the locomotor central pattern generators with adaptive processes needed to generate appropriate synaptic inputs to motoneurons during different motor tasks.

Keywords: motoneurons, muscarinic-dependent-short-term potentiation/modulation of synaptic transmission, commissural cholinergic interneurons

\section{INTRODUCTION}

Locomotion requires precise temporal and spatial activation patterns of muscles between the two sides of the body and among antagonistic muscles. In the ventral part of the spinal cord, the central pattern generators (CPGs) generate both locomotor rhythm and pattern. Electrophysiological, anatomical and, more recently, genetic approaches have provided detailed descriptions of the different interneuron (IN) subtypes that are components of the locomotor CPGs (for review see for example: Grillner et al., 2008; McCrea and Rybak, 2008; O'Donovan et al., 2008; Roberts et al., 2008; Garcia-Campmany et al., 2010; Kiehn, 2011). In the rodent spinal cord, locomotor CPG INs located in lamina VII, VIII, and $\mathrm{X}$ have been categorized into five different classes (V0, V1, V2, V3, and Hb9) based on transcription factor expression (GarciaCampmany et al., 2010). Whereas the locomotor rhythm (or timing) generation relies on ipsilaterally projecting glutamatergic INs, interneurons whose axons cross the ventral commissure, termed commissural INs (CINs), originate the reciprocal coordination between the left and right sides of the spinal cord (for review see Kiehn, 2006).

In addition to the INs involved in the fast synaptic transmission within the locomotor CPG, an intrinsic source of neuromodulation has been described in the ventral spinal networks. Partition cells and some lamina $\mathrm{X}$ neurons represent two different types of cholinergic INs (Michael et al., 1997) that have been shown to be part of the locomotor CPG (Huang et al., 2000; Zagoraiou et al., 2009). Amongst lamina X cholinergic neurons clustered around the central canal, a V0 IN subtype expressing the paired-like homeodomain protein Pitx2 has been recently described (Zagoraiou et al., 2009; Enjin et al., 2010).

The second type of cholinergic INs, the partition cells, are medium to large size neurons located in lamina VII from the central gray to the latter edge of the spinal cord (Phelps et al., 1984). A portion of these neurons sends their axons through the spinal cord midline and can then be referred to as cholinergic CINs (Phelps et al., 1984; Huang et al., 2000; Stepien et al., 2010). Partition cells are active during fictive locomotion in cats (Huang et al., 2000) and retrograde labeling with a restricted rabies virus recently highlighted the ipsilateral and bilateral monosynaptic connectivity between partition cells and lumbar motoneurons in the rodent spinal cord (Stepien et al., 2010).

Numerous studies have shown that cholinergic neuromodulation plays a major role in spinal motor systems. Acetylcholine (Ach) has been shown to inhibit the synaptic transmission between sensory afferents and motoneurons (for example see Kurihara et al., 1993). Moreover, in the isolated spinal cord preparation of newborn rodents, a bath application of Ach and/or a cholinesterase blocker triggers activities characterized by an alternating left-right firing pattern and synchronized activity between the extensor and the flexor motoneurons (Smith et al., 1988; Cowley and Schmidt, 1994; Anglister et al., 2008). At the cellular level, the activation of muscarinic receptors has been reported to increase motoneuronal excitability (Alaburda et al., 2002) via M2 receptors and inhibition of $\mathrm{Ca}^{2+}$-activated potassium channels (Miles et al., 2007). Interestingly, it has been recently showed that the genetic inactivation of all of the cholinergic V0 neurons impairs the modulatory 
control of extensor burst amplitude during swimming, suggesting an important role for these neurons in task-related modulation (Zagoraiou et al., 2009).

To further describe the cholinergic system in the locomotor CPG, we provide, in the present study, the first description of the connectivity between cholinergic CINs and motoneurons using electrical stimulations of the ventral commissure and show that the muscarinic commissural transmission, originating from both lamina $\mathrm{X}$ and partition cells, strengthens the commissural glutamatergic inputs in motoneurons in a pathway-specific manner.

\section{MATERIALS AND METHODS ETHICAL STANDARDS}

Experiments were performed using 104 Sprague-Dawleys rats (5to 12-day-old) bred in our laboratory. All animal procedures were performed with the approval of the Institutional Animal Care and the Use committee of Bordeaux University.

\section{SPINAL CORD SLICE PREPARATION}

The animals were anesthetized using isofluorane until reflexes were lost, then decapitated. The skin of the back was removed, and a laminectomy was performed to expose and remove the spinal cord in ice-cold sucrose-based saline containing the following bubbled with $95 \% \mathrm{O}_{2}-5 \% \mathrm{CO}_{2}: 2 \mathrm{mM} \mathrm{KCl}, 0.5 \mathrm{mM} \mathrm{CaCl}_{2}, 7 \mathrm{mM}$

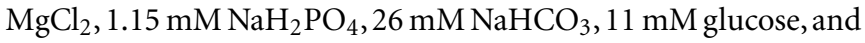
$205 \mathrm{mM}$ sucrose. Transverse slices $(350 \mu \mathrm{m})$ of the lumbar part of the cord were cut with the aid of a vibroslicer and transferred to a holding chamber. Slices were allowed to recover in oxygenated $\left(95 \% \mathrm{O}_{2}-5 \% \mathrm{CO}_{2}\right)$ artificial cerebrospinal fluid (aCSF) containing the following for at least $1 \mathrm{~h}$ at $30^{\circ} \mathrm{C}$ prior recordings: $130 \mathrm{mM} \mathrm{NaCl}, 3 \mathrm{mM} \mathrm{KCl}, 2.5 \mathrm{mM} \mathrm{CaCl}_{2}, 1.3 \mathrm{mM} \mathrm{MgSO}_{4}$, $0.58 \mathrm{mM} \mathrm{NaH}_{2} \mathrm{PO}_{4}, 25 \mathrm{mM} \mathrm{NaHCO}_{3}$, and $10 \mathrm{mM}$ glucose.

\section{WHOLE-CELL RECORDINGS AND COMMISSURAL STIMULATION}

Whole-cell current or voltage-clamp recordings from motoneurons, identified by their relatively large size in lamina IX in neonatal rat spinal cord slices, were made under visual control with a Multiclamp 700B amplifier. Recording glass microelectrodes (4$7 \mathrm{M} \Omega$ ) were filled with the following with a $\mathrm{pH}$ of 7.3: $120 \mathrm{mM}$ K-gluconate, $20 \mathrm{mM} \mathrm{KCl}, 0.1 \mathrm{mM} \mathrm{MgCl}_{2}, 1 \mathrm{mM}$ EGTA, $10 \mathrm{mM}$ HEPES, $0.1 \mathrm{mM} \mathrm{CaCl}_{2}, 0.1 \mathrm{mM}$ GTP, $0.2 \mathrm{mM}$ cAMP, $0.1 \mathrm{mM}$ Leupeptin, $77 \mathrm{mM}$ D-Mannitol, and $3 \mathrm{mM} \mathrm{Na}$-ATP. For post hoc localization and identification of the recorded cells, $0.02 \%$ biocytin was added to the intracellular solution. Electrical stimulation of the axons crossing through the ventral commissure was conducted through bipolar tungsten electrodes (see Figure 1A; tip separation: 75-100 $\mu \mathrm{m}$, stimulation duration: $500 \mu \mathrm{s}$, stimulation intensities

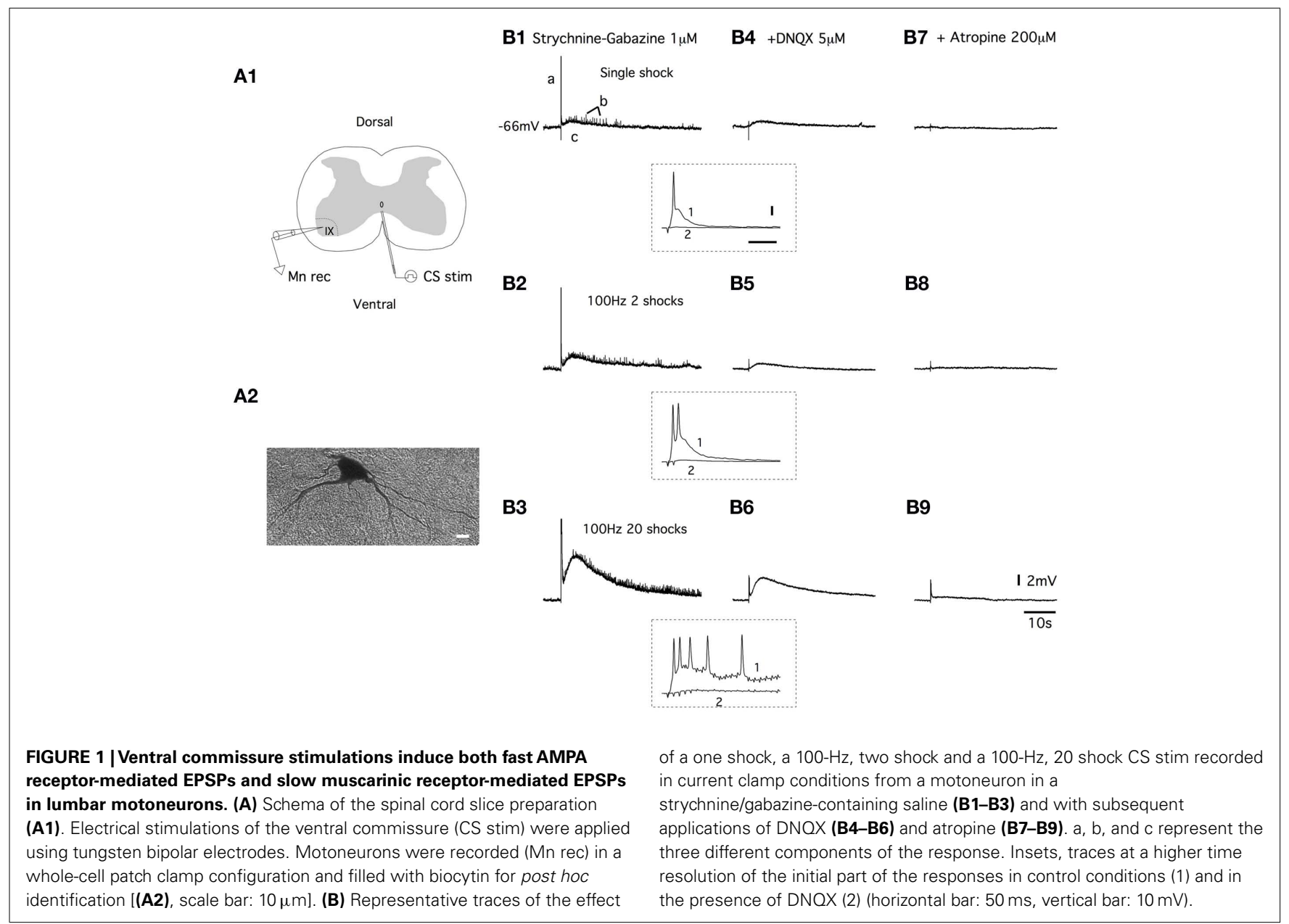


ranging from 20 to $400 \mu \mathrm{A}$ ). To record monosynaptic excitatory postsynaptic potentials (EPSPs) or currents (EPSCs), the polysynaptic transmission was depressed in all of the experiments using a high cation-containing aCSF with $7.5 \mathrm{mM} \mathrm{CaCl}_{2}$ and $8 \mathrm{mM} \mathrm{MgSO}_{4}$. Strychnine and gabazine $(1 \mu \mathrm{M})$ were also added throughout the experiments to block the glycinergic and $\mathrm{GABA}_{\mathrm{A}}$ receptor activation, respectively. All experiments were performed at room temperature $\left(25^{\circ} \mathrm{C}\right)$. Oxotremorine $(500 \mu \mathrm{M})$ was applied on motoneurons with a fast exchanging solution system. Three applications ( $2-5 \mathrm{~s}$ long) separated by $6 \mathrm{~min}$ were performed, and the mean value of the oxotremorine-induced depolarization was computed.

\section{IN VITRO SPINAL CORD PREPARATION AND COMMISSURAL STIMULATION}

Spinal cords from P4 to P6 rats were collected as previously described in the spinal cord slice preparation section and pinned down with the ventral side upward. The ventral spinal artery, which enters the ventral median fissure, was removed at the L5 level. The pia was then opened and a bipolar tungsten electrode was vertically inserted to stimulate the ventral commissure (Figure 10A). Locomotor-like activity was induced using a mixture of $N$-methyl-D-L-aspartate (NMA, $16 \mu \mathrm{M})$ and serotonin $(5 \mathrm{HT}, 16 \mu \mathrm{M})$. Motor activity was recorded from the rL2, rL5, and IL5 ventral roots using stainless steel electrodes insulated with Vaseline (dots in Figure 10A). Locomotor parameters were computed using a program developed in Matlab (Mathworks). The coupling between left and right and flexor-extensor activities was analyzed using circular statistics on at least 20 locomotor bursts using the Oriana software (Kovach Computing Services). The results of this analysis gave the mean vector of the phase distribution and its length $r$ (ranging from 0 to 1 ), indicating the strength of the mean. The L2 and L5 phase values have been plotted on a circle representing the interval of possible phases from 0 to $360^{\circ}$. Phase values 0 and $360^{\circ}$ reflect synchrony, and a phase value of $180^{\circ}$ reflects alternation. The mean phase $\Phi$ was indicated by the direction of the vector originating from the center of the circle. A Rayleigh test ( $Z$ ) was used to assess the significance of the coupling. A multisample testing was performed using the WatsonWilliams $F$-tests to assess the similarity between the mean vectors before and after the pharmacological treatment.

\section{RETROGRADE LABELING AND IMMUNOCHEMISTRY}

Transverse slices $(350 \mu \mathrm{m})$ of the L3-L5 lumbar part of the cord from two P8-9 rats were prepared as for the patch clamp recording experiments. Because targeted cells have been shown to be located in the vicinity of the ventral commissure (Zagoraiou et al., 2009; Enjin et al., 2010; Stepien et al., 2010), a small amount of Texas Red dextran amine (TxR; $3000 \mathrm{kDa}$ ) was applied with an insect pin ventrally to the central canal to prevent a massive labeling of surrounding areas. The slices were then kept for $6-8 \mathrm{~h}$ in the dark in oxygenated aCSF at $30^{\circ} \mathrm{C}$. After this migration period, the slices were immerged in $4 \%$ paraformaldehyde in phosphate-buffered saline (PBS) overnight at $4^{\circ} \mathrm{C}$. After cryoprotection, $30 \mu \mathrm{m}$ cryosections were mounted on slides and were processed for Choline AcetylTransferase (ChAT) immunodetection. The primary antibody used was a goat anti-ChAT antibody (1:100, Millipore) revealed with an Alexa fluor 488 donkey anti-goat secondary antibody (1:500). Images of TxR and ChAT immunopositive cells were obtained using a Nikon AZ100M microscope. Drawings of each individual section were realized and superimposed on a normalized spinal cord section to illustrate the position and size of the different double-labeled neurons.

\section{DATA ANALYSIS}

All data are expressed as means \pm SEM in the text and in the figures. The asterisks in the figures indicate positive significance levels, and the numbers in or above the histogram bars refer to the number of neurons examined.

\section{RESULTS VENTRAL COMMISSURAL STIMULATIONS TRIGGER SLOW MUSCARINIC EPSPS IN LUMBAR MOTONEURONS}

The application of electrical stimulation to the ventral commissure (CS stim; Figure 1A1) generated complex depolarizations in motoneurons (Figure 1A2) that were recorded in current clamp conditions. In all the motoneurons tested, the application of a single shock CS stim triggered responses with three distinguishable components (a, b, and c; Figure 1B1). The first component consisted of a fast EPSP that reached the spike threshold (a in Figure 1B1 and trace 1 in inset in Figure 1B1-B4). This response was followed by fast EPSPs of small amplitudes (b) superimposed on a long-lasting depolarization (c; Figure 1B1). When $100 \mathrm{~Hz}$ stimulation trains of 2 (Figure 1B2) or 20 shocks (Figure 1B3) were delivered to the ventral commissure, the pattern of the responses remained, but the amplitude of the depolarization was strongly increased. Using $100 \mathrm{~Hz}$ stimulations, we observed an increase in the number of both spikes generated in the first part of the response (traces 1 in inset in Figure 1B1-B6) and small amplitude EPSPs accompanied with a significant enhancement of the long-lasting EPSP amplitude (Figure 1B2,B3). Both spikes (traces 2 in inset in Figure 1B1-B6) and small amplitude EPSPs were abolished in the presence of the AMPA/kainate receptor antagonist DNQX $(5 \mu \mathrm{M})$, whereas the long-lasting EPSP persisted (Figure 1B4-B6). The subsequent bath application of the muscarinic receptor antagonist atropine $(200 \mu \mathrm{M})$ almost completely suppressed the DNQX-insensitive part of the response for all of the stimulation modalities used (Figure 1B7-B9). These results show that the CS stimulation induced both fast AMPA receptor-mediated EPSPs (AMPA-EPSPs) and slow muscarinic receptor-mediated EPSPs (muscEPSPs) in lumbar motoneurons.

In strychnine/gabazine/DNQX-containing aCSF, we examined the dose-dependency of the atropine block on the commissural muscEPSP induced with a $100 \mathrm{~Hz}, 20$ shock CS stim. As shown in Figure 2A1 and in the plot in Figure 2A2, bath applications of $10 \mu \mathrm{M}$ atropine significantly reduced the muscEPSP amplitude. Raising the atropine concentration progressively to $200 \mu \mathrm{M}$ led to the complete inhibition of muscEPSP (Figure 2A1,A2). The effects of atropine were completely or partially reversed after a 30-min washout period (Figure 2A1,A2).

In a next step, we investigated the efficiency of different CS stim modalities in inducing muscEPSPs. For this purpose, we varied the stimulation frequency and the number of shocks delivered to the ventral commissure. As exemplified in Figure 2B1, 


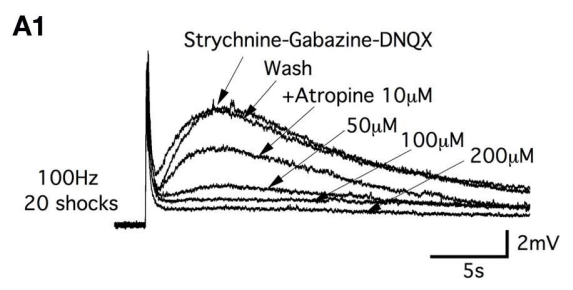

B1

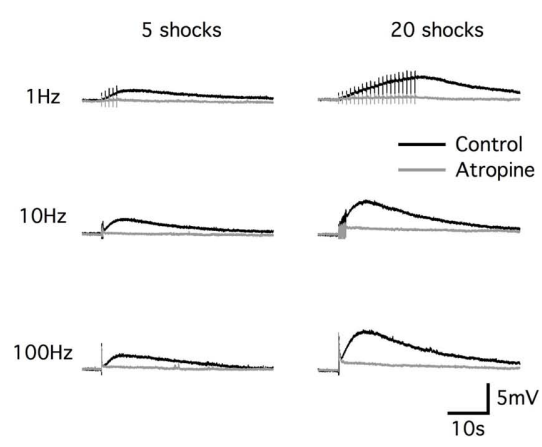

FIGURE 2 | Atropine sensitivity of muscarinic receptor-mediated EPSPs and CS stimulation parameters. (A) Superimposed muscarinic (musc) EPSPs induced by a CS stim in the presence of different atropine concentrations (A1). Plot of the mean muscEPSP amplitude as a function of the atropine concentration (A2). Washout period: $30 \mathrm{~min}$. (B)

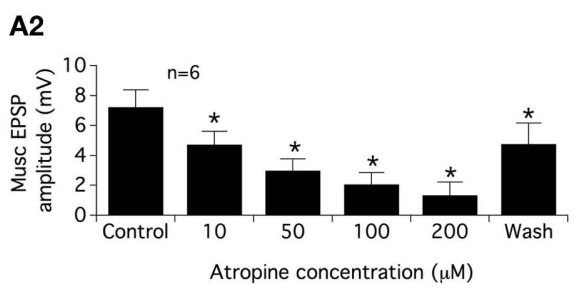

B2

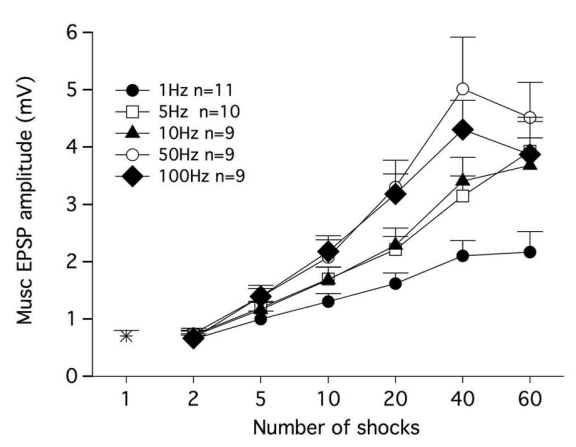

Representative traces of muscEPSP induced with different CS stimulation protocols (B1) in control conditions (black traces) and in the presence of atropine (200 $\mu$ M, gray traces). (B2) Summary plot of the mean muscEPSP amplitude as a function of the number of shocks and frequency of the CS stim. we first observed that regardless of the frequency, a long-lasting atropine-sensitive EPSP was generated in motoneurons. The muscEPSP amplitude appeared to be dependent on both the CS stim frequency and the number of shocks in the stimulation trains. Increasing the CS stim frequency and shock number in the trains boosted the muscEPSP amplitude until maximum muscEPSP amplitude values were obtained using 50 or $100 \mathrm{~Hz}$ trains of 40 shocks (Figure 2B2). Raising the shock number up to 40 in 50 or $100 \mathrm{~Hz}$ trains caused a decrease of muscEPSP amplitudes (Figure 2B2). Therefore, in all of the following experiments, muscEPSPs were studied using a CS stim of $100 \mathrm{~Hz}$ trains with 20 shocks (CS high-frequency stimulation; CS HFS) unless otherwise specified.

To examine the ionic basis of the muscEPSPs, the variations of the motoneuron input membrane resistance were computed using $50 \mathrm{pA}$ hyperpolarizing current injections applied at $0.8 \mathrm{~Hz}$ and superimposed with a CS HFS (Figure 3A1). As shown on the representative trace in Figure 3A2, muscEPSPs were accompanied by a small but significant increase in the input membrane resistance that was $72.4 \pm 10 \mathrm{M} \Omega$ in control conditions and $81.1 \pm 12 \mathrm{M} \Omega$, $n=11,5 \mathrm{~s}$ after the CS HFS (paired $t$-test, $p=0.004$ ). Acetylcholine acting at muscarinic receptors reduces a slowly activating voltage-regulated $\mathrm{K}^{+}$current, the $\mathrm{M}$-current, in numerous systems (for review see Brown and Passmore, 2009). To assess whether muscEPSPs are sustained by the M-current in lumbar motoneurons, we tested the effects of the M-current antagonist XE991 on CS HFS-induced responses. In voltage-clamp conditions, the superfusion of $10 \mu \mathrm{M}$ XE991 in motoneurons held at $-60 \mathrm{mV}$ induced an inward current with a mean amplitude of $-90 \pm 19 \mathrm{pA}, n=10$ (data not shown). When stimulation trains $(100 \mathrm{~Hz}, 20$ shocks) were applied to the ventral commissure in current clamp conditions, in the presence of XE991, we observed first, an enhancement of the neuronal excitability as shown by the increase in the number of spikes expressed in the first part of the CS HFS-induced responses (compare black and gray traces in Figure 3B2) and second, a significant and reversible decrease of muscEPSP amplitudes compared to control conditions (Figure 3B1-B5). To assess the specificity of the XE991 blockade, we tested the effects of two other $\mathrm{K}^{+}$channel blockers: 4 -aminopyridine (4-AP; $50 \mu \mathrm{M}$ ) and apamin $(100 \mathrm{nM})$ on muscEPSPs. We observed that both compounds increased the motoneuron excitability (Figure 3B4, data not shown for 4-AP) but, in contrast to XE991, significantly increased muscEPSP amplitudes (Figures 3B3-B5). The subsequent bath application of XE991 in the presence of 4-AP or apamin $(n=5)$ further increased the motoneuron excitability (Figure 3B4, trace 3 ) and led to the complete inhibition of muscEPSP expression. Altogether, these results show that the M-current is tonically active in lumbar motoneurons and that muscEPSPs are linked to the closure of the M-current in these neurons.

Muscarinic receptors belong to the large family of $G$ proteincoupled receptors. Five different subtypes of muscarinic receptors numbered from 1 to 5 have been identified so far. To assess their respective role in the commissural muscarinic (CM) transmission in lumbar motoneurons, we supplemented the strychnine/gabazine/DNQX-containing aCSF with various muscarinic receptor subtype antagonists, pirenzepine (M1 preferring), AF-DX116 (M2 preferring), 4-DAMP (M3 preferring) or tropicamide (M4 preferring), and we used the CS stim protocol of 


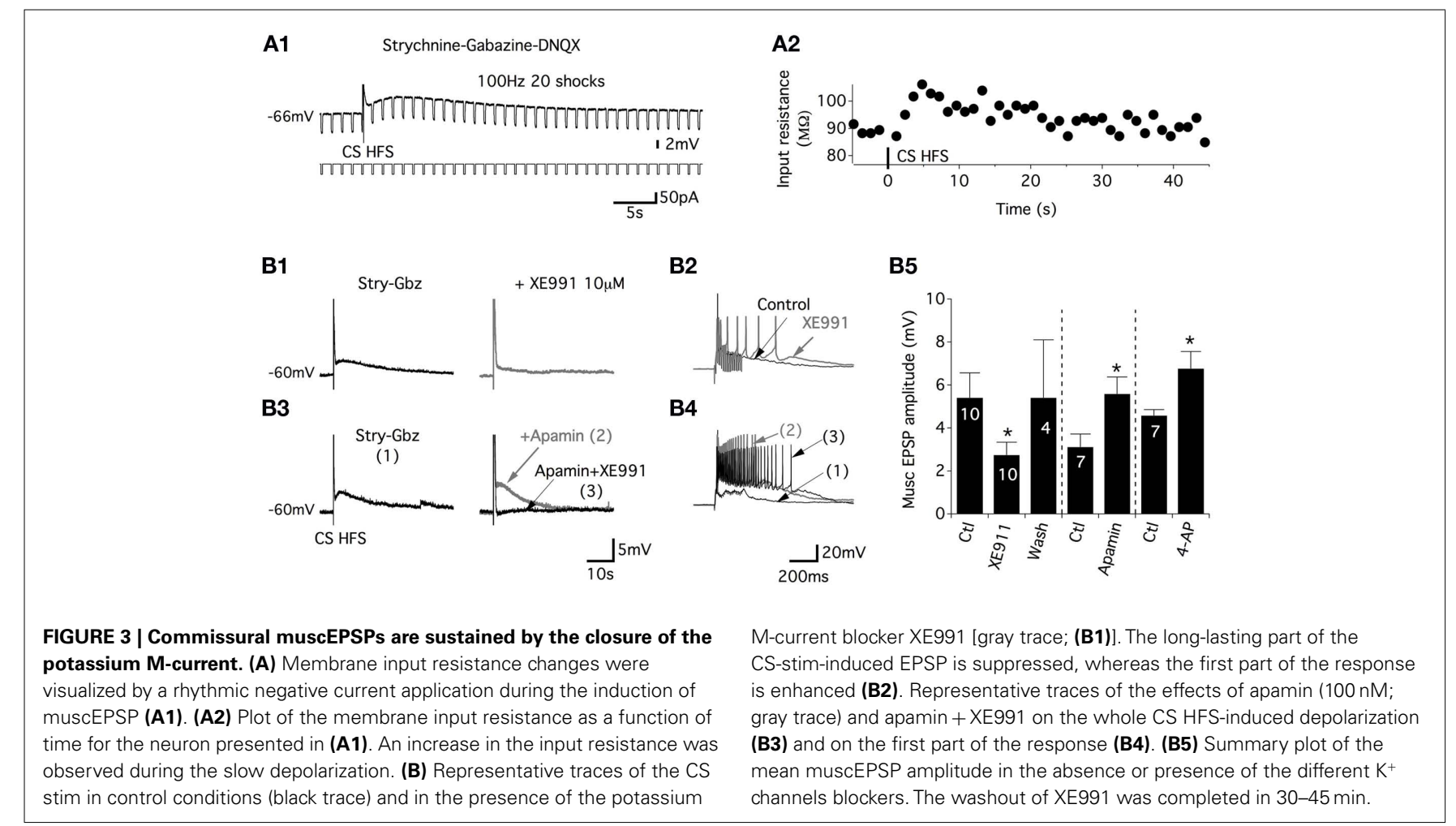

20 shocks at $100 \mathrm{~Hz}$. The M5 muscarinic receptor is the most recent member of the muscarinic acetylcholine receptor family to be cloned, and to date, selective commercial ligands that can inhibit this receptor type are missing.

The muscEPSP amplitude and time constant were then analyzed. First, we observed that $10 \mu \mathrm{M}$ of the M2 or M4 antagonist failed to alter the muscEPSP parameters (Figure 4A3,A4). Nevertheless, an application of $100 \mu \mathrm{M}$ tropicamide significantly decreased the amplitude and increased the time constant of the muscEPSP in a reversible manner (Figure $\mathbf{4 A 1}, \mathbf{A 3}, \mathbf{A 4}$ ). In the same way, motoneurons superfused with an aCSF containing $50 \mu \mathrm{M}$ of the M2 preferring antagonist AF-DX116 expressed muscEPSPs with significantly reduced amplitudes and time constants that tended to be longer (Figure 4A3,A4). Commissural muscEPSPs were, in contrast, insensitive to the superfusion of the M1 antagonist pirenzepine for any concentration that we tested (Figure 4A3,A4). Finally, we investigated the potential role of the M3 muscarinic receptor in the cholinergic commissural transmission. Although $1 \mu \mathrm{M}$ 4-DAMP caused no significant effects, raising the concentration to $10 \mu \mathrm{M}$ led to a significant increase in both the amplitude and the time constant of the muscEPSPs induced by CS HFS (Figure 4A2-A4). These effects were reversed after a 30-45-min washout period.

The data reported above could have resulted from intermingled pre- and post-synaptic effects of the muscarinic receptor antagonists on cholinergic commissural transmission. To assess the postsynaptic actions of the muscarinic antagonists, these compounds were tested in motoneurons synaptically isolated with tetrodotoxin (TTX, $0.5 \mu \mathrm{M}$ ). To stimulate the muscarinic receptors expressed in the motoneuron membrane, oxotremorine, a wide range muscarinic receptor agonist, was applied on the lumbar motoneurons (see Materials and Methods). Because muscarinic receptor activation has been shown to modulate calcium channels (see for example: Zhang et al., 2009), these experiments were performed in a $2 \mathrm{mM} \mathrm{Mn}^{2+}$-containing aCSF without $\mathrm{Ca}^{2+}$. In these experimental conditions, short-lasting applications of oxotremorine $(500 \mu \mathrm{M}, 2-3 \mathrm{~s})$ generated very long-lasting depolarizations in motoneurons (Figure 4B1,B2) that were significantly depressed by XE991 (Figure 4B3). A dose-dependent inhibition of the oxotremorine-induced depolarization was observed with the four different muscarinic antagonists tested (see examples in Figure 4B1,B2). These depressing effects were observed in concentration ranges from 1 to $10 \mu \mathrm{M}$ for pirenzepine, AF-DX 116 and tropicamide and from 0.5 to $1 \mu \mathrm{M}$ for 4-DAMP (Figure 4B3). Altogether these data indicate that the lumbar motoneurons express the M1, M2, M3, and M4 muscarinic receptors and that the cholinergic commissural transmission is sustained by the activation of M2, M4, and M3 receptors (see Discussion).

\section{MUSCARINIC RECEPTOR ACTIVATION INDUCES A SHORT-LASTING SYNAPTIC ENHANCEMENT OF THE GLUTAMATERGIC TRANSMISSION}

For the next step, we sought to determine whether cholinergicglutamatergic interactions exist in the ventral motor spinal cord as described, for example, in the hippocampus (Fernandez de Sevilla et al., 2008). In the voltage-clamp mode, in motoneurons held at $-60 \mathrm{mV}$, a one shock CS stim evoked commissural EPSCs (Figure 5A1), that were abolished by the AMPA/kainate antagonist DNQX (10 $\mu \mathrm{M}$, data not shown; com-AMPA-EPSCs). After a 10$15-$ min control period of $0.3 \mathrm{~Hz}$ stimulations, a $100-\mathrm{Hz}, 20$ shock CS stim was applied in the current clamp mode. When returned to 


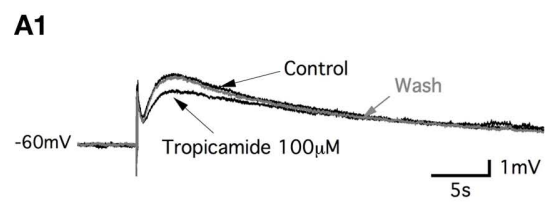

A3

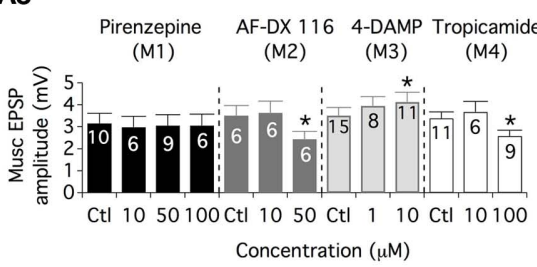

B1
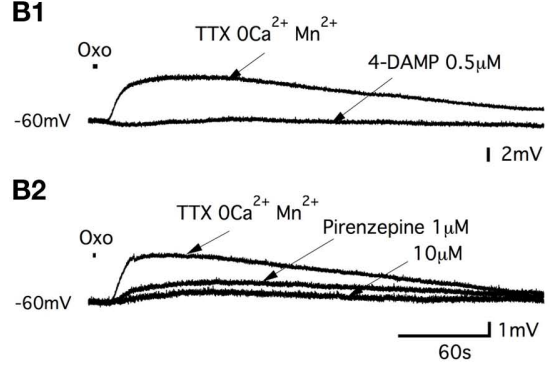

FIGURE 4 | Commissural muscEPSPs involve the activation of M2, M3, and M4 muscarinic receptors. (A) Representative traces of the effects of the M4-preferring antagonist, tropicamide (A1), and those of the M3-preferring antagonist, 4-DAMP (A2), on muscEPSP induced in control conditions (strychnine/gabazine/DNOX-containing aCSF) and after the washout of the drug (30-45 min). Summary plots of the mean muscEPSP amplitude (A3) and time constant (A4) as a function of the muscarinic

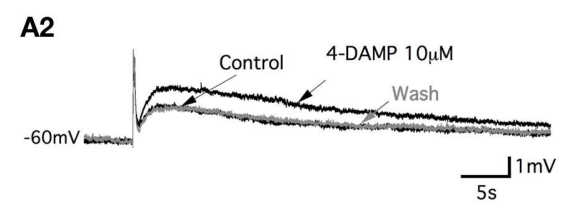

A4

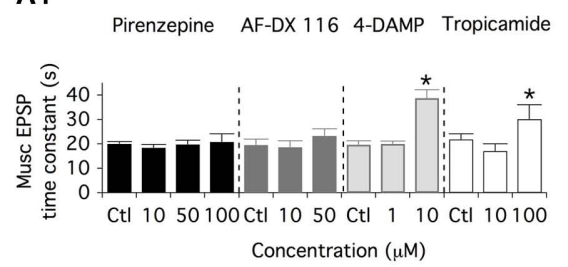

B3

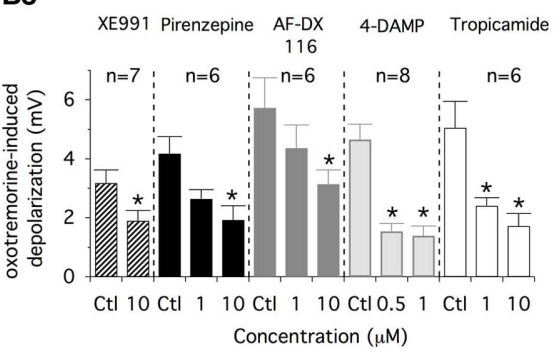

antagonist concentration. (B) Traces illustrating the actions of 4-DAMP (B1) and pirenzepine (B2) on depolarizations induced by short-lasting applications of oxotremorine (oxo, $500 \mu \mathrm{M}$ ) in motoneurons synaptically isolated in a $\mathrm{TTX} / 2 \mathrm{mM} \mathrm{Mn}{ }^{2+}$-containing aCSF without $\mathrm{Ca}^{2+}$. (B3) Summary plot of the mean amplitude of the oxotremorine-induced depolarization in the absence or presence of XE991 and as a function of the different muscarinic antagonist concentrations tested. the voltage-clamp mode, a short-term potentiation (STP) of the com-AMPA-EPSC area was observed (Figure 5A1). Computed for the entire population of motoneurons tested, the mean comAMPA-EPSC area was amplified by $40 \pm 5 \%$ immediately after a CS HFS compared with the mean area of the five last baseline control EPSCs $(n=26$; Figure 5A2). This enhancement was observed in all of the motoneurons tested and dissipated within approximately $500 \mathrm{~s}$ (Figure 5A2). To test whether the activation of the muscarinic receptors contributes to the com-AMPA-EPSC STP, a first CS HFS was applied in control saline (black traces in Figure 5B) to check that com-AMPA-EPSC STP could be elicited in the tested motoneurons. The subsequent bath application of atropine caused a significant decrease of the baseline com-AMPAEPSC area (Mann-Whitney test, $p=0.005, n=9$; Figure 5B1,B2). In the presence of the muscarinic receptor antagonist, a second CS HFS completely failed to induce a com-AMPA-EPSC STP (Figure 4B1, gray traces). For the population of motoneurons tested in these experimental conditions, the mean com-AMPAEPSC area amplification was $36 \pm 6 \%$ in normal aCSF (black dots in Figure 5B2), $4 \pm 6 \%$ in atropine-containing aCSF (gray dots in Figure $5 \mathrm{~B} 2 ; n=9)$ and $31 \pm 6 \%(n=6)$ after a full washout of atropine (45-60 min, data not shown).

As previously shown, one shock CS stimulations trigger both AMPA- and muscarinic EPSPs (Figure 1B). To verify whether the muscarinic-dependent STP (musc-STP) we observed is linked to an increase in the glutamatergic part of the CS stim-induced responses and not in the muscarinic one, the effects of CS HFS were tested in the presence of DNQX to isolate the CM inputs (Figure 5C1). Due to the small amplitude and long duration of one shock CS stim-induced muscEPSPs, these experiments were performed in current clamp conditions and the stimulation frequency used to generate baseline muscEPSPs was set to $1 \mathrm{~Hz}$. In these conditions, we observed that CS HFS-induced a small depression of the muscEPSP area (Figure 5C1,C2). These results suggest that the release of acetylcholine by commissural cholinergic axons triggers a musc-STP of the commissural glutamatergic transmission.

In a next step, we investigated whether the musc-STP of comAMPA-EPSCs is linked to the $100-\mathrm{Hz}$ stimulation or whether other stimulation protocols that had been previously described as efficient means to evoke muscarinic responses in motoneurons (Figure 2B) could modify the synaptic strength of commissural glutamatergic inputs. Based on the results presented in Figure 2B, four different stimulation paradigms were tested: a low-frequency stimulation ( $1 \mathrm{~Hz}, 40$ shocks, LFS; Figure 6A), two "medium"-frequency stimulations $(10 \mathrm{~Hz}, 20$ and 40 shocks, MFS; Figures 6B,C) and a HFS (50 Hz, 20 shocks; Figure 6D). All four stimulation modalities triggered a com-AMPA-EPSC STP (Figure 6). The area of the com-AMPA-EPSCs was, significantly, boosted by $15 \pm 8 \%$ using a CS LFS ( $n=6$; Figure 6A), $16 \pm 7 \%$ with a 20 shock MFS ( $n=5$; Figure $6 B), 37 \pm 16 \%$ with a 40 shock 


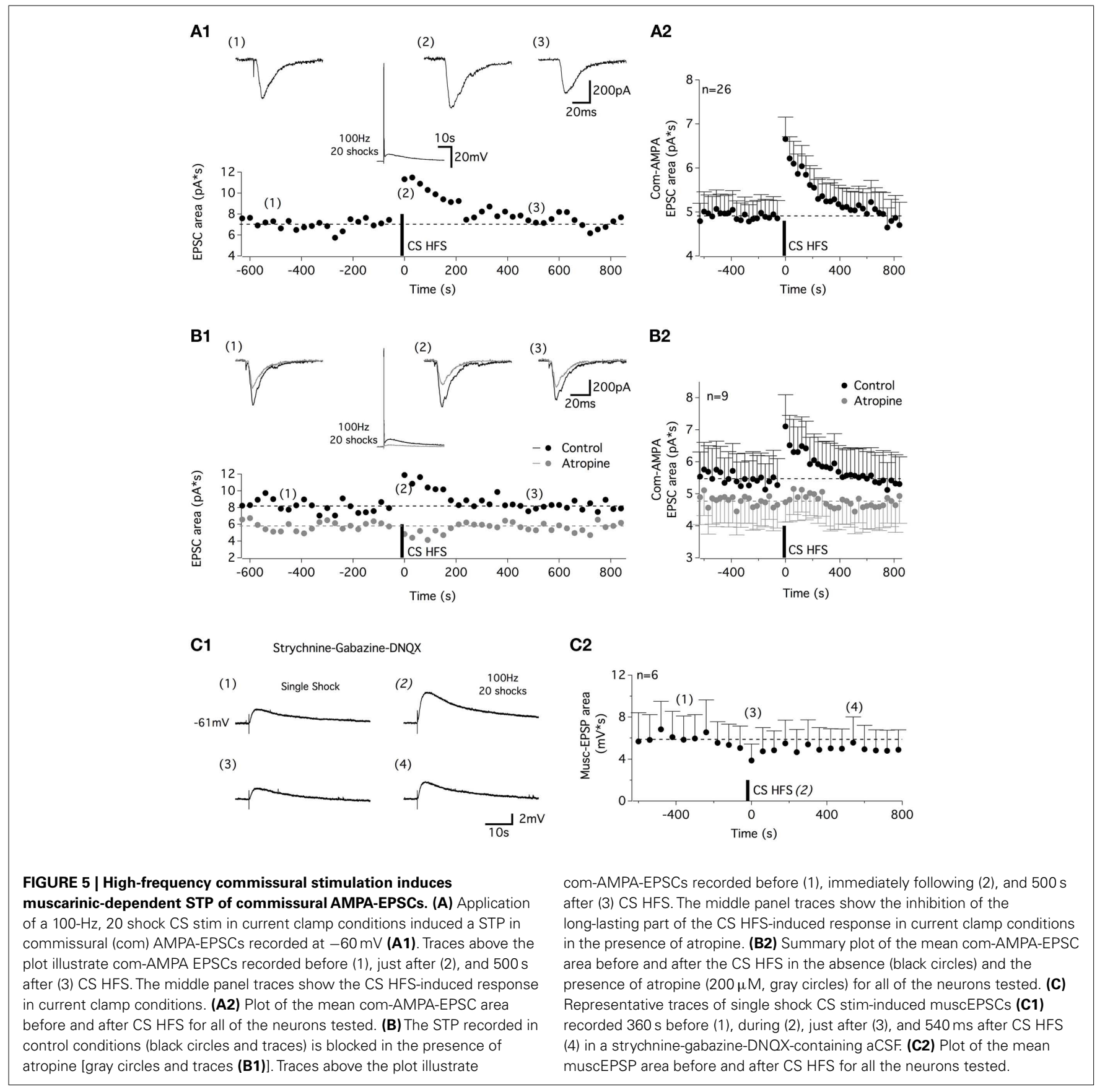

MFS ( $n=5$; Figure $6 \mathrm{C}$ ), and $37 \pm 9 \%$ by a $50-\mathrm{Hz} \mathrm{HFS}(n=6$; Figure 6D). Regardless of the stimulation protocol used, the expression of the com-AMPA-EPSC STP was completely blocked in the presence of atropine ( $n=5$, data not shown).

The effects we observed from the application of the M-current antagonist, XE991 and M3 receptor antagonist, 4-DAMP on muscEPSPs (Figure 4) prompted us to examine their potential role in the muscarinic-dependent potentiation of the com-AMPAEPSCs. In addition to inhibiting the atropine-sensitive part of the CS HFS-induced depolarization (middle panel in Figure 7A1, see also Figure 3), XE991 significantly modified the baseline
com-AMPA-EPSC area from $4.1 \pm 0.1 \mathrm{pA}^{*} \mathrm{~s}$ in control conditions to $3.3 \pm 0.1 \mathrm{pA}^{*} \mathrm{~s}$ in the presence of the M-current antagonist (Mann-Whitney test, $p<0.001, n=11$; Figure 7A2). In contrast, the magnitude of the musc-STP was unchanged in a XE991-containing aCSF compared to control conditions (two-way ANOVA, $p=0.4$; Figure 7A2). The mean com-AMPA-EPSC area was enhanced by $49 \pm 11 \%$ in the absence and $57 \pm 16 \%(n=11$ neurons) in the presence of XE991. In a next step, we analyzed the effect of a 20 shock $100 \mathrm{~Hz}$ Cs stim before and after the superfusion of the M3-preferring antagonist 4-DAMP. At a concentration $(10 \mu \mathrm{M})$ that has been shown to enhance the muscEPSP amplitude 

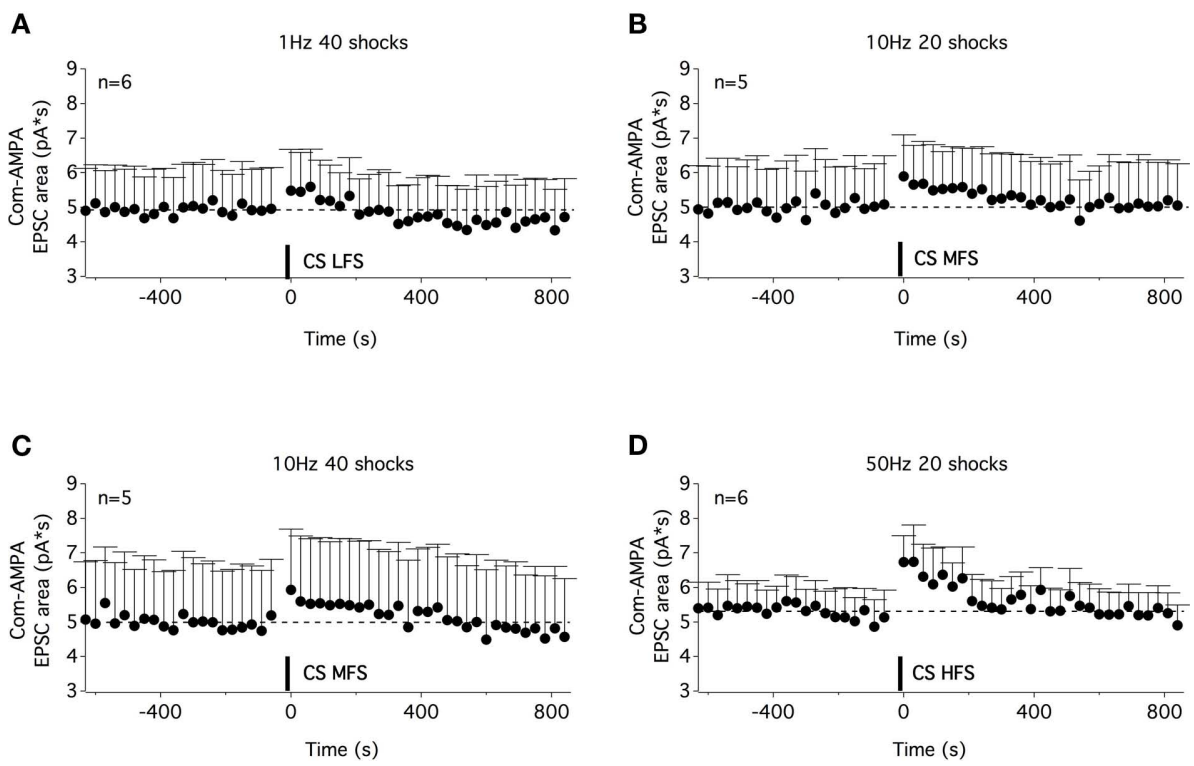

FIGURE 6 | A STP of commissural AMPA-EPSCs could be induced with different stimulation paradigms of the ventral commissure. Time courses of the com-AMPA-EPSC area before and after a low-frequency stimulation of the ventral commissure [CS LFS (A)], a medium-frequency stimulation (MFS) of 20 shocks (B), of 40 shocks (C), and a high-frequency stimulation (D). A STP of com-AMPA-EPSC could be observed with the different stimulation protocols used.

and duration (middle panel in Figure 7B1, see also Figure 4A), 4-DAMP had no significant effect on either the baseline comAMPA-EPSC area and the STP extent that were $5.1 \pm 0.1 \mathrm{pA}^{*} \mathrm{~s}$ and $28 \pm 8 \%$ in control conditions and $4.9 \pm 0.1 \mathrm{pA}^{*} s(p=0.2)$ and $27 \pm 12 \%$ ( $p=0.8, n=8$ neurons) in 4-DAMP-containing aCSF, respectively (Figure 7B2).

The question now arises as to whether all of the glutamatergic pathways synapsing on lumbar motoneurons are potentiated by the CS HFS or whether this synaptic enhancement is restricted to specific glutamatergic afferents. The ventral part of lamina VII and laminae VIII and IX contain interneurons that are assumed to be part of the locomotor CPG. Among the various classes of interneurons that have been described, the ipsilateral and contralateral glutamatergic neurons located in lamina VII have been shown to project monosynaptically to lumbar motoneurons (for review see Kiehn, 2011). To address the question of the pathway specificity of the musc-STP, we then chose to test the impact of a CS HFS on inputs arising from the ipsilateral or contralateral lamina VII. For this purpose, one stimulating electrode was placed in the ipsi- or contralateral ventral part of the lamina VII to induce glutamatergic ipsi or contra lamina VII EPSCsin motoneurons, respectively, and a second stimulating electrode was positioned on the ventral commissure as was done previously (Figures 8A1,B1). Under our experimental conditions (i.e., in a strychnine/gabazine/high cation-containing saline), a one shock stimulation delivered every $30 \mathrm{~s}$ to the ipsi lamina VII induced stable EPSCs. The subsequent application of a CS HFS failed to modify the ispi lamina VII EPSC area $(0.5 \pm 6 \%, n=8$; Figure 8A2), whereas a STP of the com-AMPA-EPSCs could be induced in the same neurons when long duration recordings could be achieved $(45 \pm 15 \%, n=5$; Figure 8A3). In contrast, when the effects of a CS HFS were tested on the glutamatergic inputs originating from the contralateral lamina VII, an atropine-sensitive-STP of the contra lamina VII EPSC area was observed (Figure 8B2). For the population of motoneurons tested, the mean contra lamina VII EPSC area was amplified by $47 \pm 12 \%, n=5$, in normal saline (black dots in Figure 8B2) and $-1 \pm 11 \%$ in an atropine-containing aCSF (gray dots in Figure 8B2; $n=5$ ).

\section{PARTITION CELLS AND LAMINA $X$ INTERNEURONS ARE THE SOURCE OF THE CHOLINERGIC COMMISSURAL TRANSMISSION IN LUMBAR MOTONEURONS}

To assess the cellular type mediating the muscarinic commissural transmission in spinal cord slices, we performed a TxR retrograde labeling of axons crossing through the ventral commissure coupled to ChAT immunoreactivity. In our experimental conditions, 14 cells retrogradely labeled and immunopositive for ChAT (TxRChAT +) were observed from 11 sections of two animals. Out of 14, 11 TxR-ChAT + neurons were located in the medial part of the lamina VII within a distance of $350 \mu \mathrm{m}$ from the lateral border of the central canal (Figures 9A-C), whereas the three remaining ones occurred in lamina X (Figures 9B,C).

\section{THE COMMISSURAL MUSCARINIC TRANSMISSION IS NOT INVOLVED IN LEFT-RIGHT OR FLEXOR-EXTENSOR COUPLING}

Commissural interneurons have been shown to be involved in left-right coordination and in flexor-extensor alternation in locomotor CPG. We therefore examined the impact of muscarinic transmission blockade on motor coupling during fictive locomotion in isolated spinal cord preparations obtained from P4 to $\mathrm{P} 6$ rats. To monitor left-right and flexor-extensor activities, the right lumbar 2 (rL2), rL5, and left L5 (lL5) ventral roots 

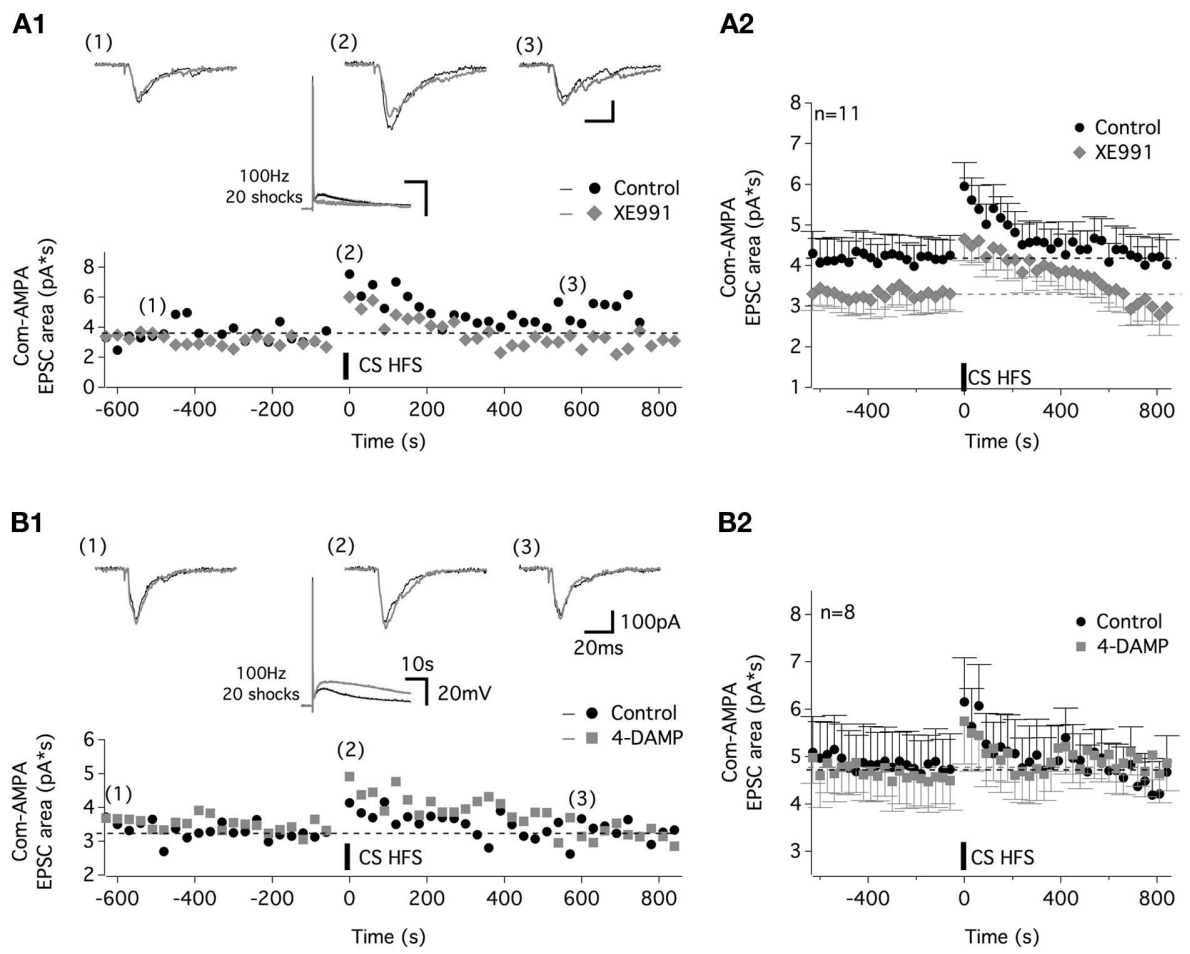

B2

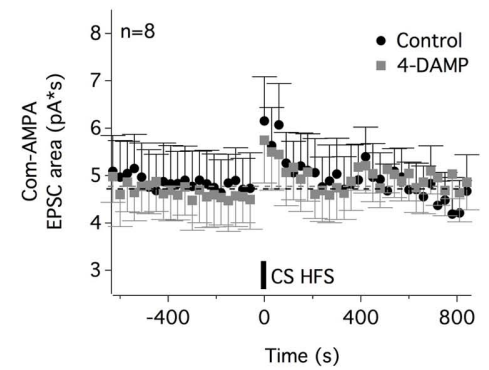

FIGURE 7 | Com-AMPA-EPSC STP is not altered by the potassium M-current antagonist, XE991, or the M3-preferring antagonist 4-DAMP. (A) Representative traces and time course of the com-AMPA-EPSC area before and after CS HFS in the control condition (black circles and traces) and in the presence of $10 \mu \mathrm{M}$ XE991 [gray lozenges and traces (A1)]. Traces above the plot illustrate the com-AMPA-EPSCs recorded before (1), immediately following (2), and $600 \mathrm{~s}$ after (3) a CS HFS. The middle panel traces show that the CS HFS-induced responses in current clamp condition without and with XE991 in the superfusion medium. (A2) The average time course of the
com-AMPA-EPSC area before and after the CS HFS in the absence (black circles) or the presence of XE991 (gray lozenges) for all of the neurons tested. (B) Representative traces and time course of the com-AMPA-EPSC area before and after CS HFS in the control condition (black circles and traces) and in the presence of $10 \mu \mathrm{M} 4-\mathrm{DAMP}$ [gray squares and traces (B1)]. Traces above the plot illustrate com-AMPA-EPSCs recorded before (1), immediately following (2), and $600 \mathrm{~s}$ after (3) CS HFS. (B2) Average time course of the com-AMPA-EPSC area before and after CS HFS in the absence (black circles) or the presence of 4-DAMP (gray squares) for all the neurons tested. were recorded (Figure 10A) with bursts in L2 and L5 corresponding to flexor and extensor activity, respectively (Kiehn and Kjaerulff, 1996). A bath application of the muscarinic antagonist atropine $(200 \mu \mathrm{M})$ in NMA-5HT-containing aCSF caused a small but significant increase in locomotor period (Figure 10B1,B2) with a mean locomotor period of $3.2 \pm 0.3 \mathrm{~s}$ in control conditions, $3.9 \pm 0.3 \mathrm{~s}$ in the presence of atropine, and $3.4 \pm 0.5 \mathrm{~s}$ after washout (30-45 $\mathrm{min}, n=7$ ). Atropine, in contrast, failed to significantly modify the burst amplitude values (Figure 10B1,B2). The circular analysis of the rL5 and IL5 bursts revealed that the left-right coupling was unaffected by the superfusion of atropine (mean $\Phi=160^{\circ}, r=0.947$ in control conditions and $186^{\circ}, r=0.925$ with atropine in the bath; Watson-Williams F-test: $p=0.064$; Figure 10B3). Moreover, the flexor-extensor coupling monitored by alternating activity between the rL2 and rL5 ventral roots and synchronicity between the rL2 and IL5 bursts was not changed in the atropine-containing saline (mean rL2-rL5 $\Phi=188^{\circ}, r=0.916$ in the absence and $160^{\circ}, r=0.92$ in the presence of atropine, Watson-Williams $F$-test: $p=0.062$; mean rL2-lL5 $\Phi=345^{\circ}, r=0.933$ in control conditions and $356^{\circ}, r=0.97$ with atropine, Watson-Williams $F$-test: $p=0.358$, $n=7)$.

\section{THE COMMISSURAL MUSCARINIC TRANSMISSION BOOSTS MOTONEURON ACTIVITY DURING FICTIVE LOCOMOTION}

The effect of a CS HFS applied at the L5 level was then assessed in the in vitro isolated spinal cord preparation. In a quiescent spinal cord, a 100-Hz, 20 shock CS HFS-induced transient activity in all the recorded ventral roots (Figure 10C1) that were not significantly modified in the presence of atropine (Figure 10C2). This result and the lack of atropine effects on the motor burst amplitude previously reported suggest that in our experimental conditions, this compound did not alter motoneuron excitability. CS HFS effects were then tested during NMA-5HT-induced fictive locomotion. Control locomotor activity was recorded during $40 \mathrm{~s}$ prior to the CS HFS. To compute motor burst changes, the burst amplitude was normalized to the mean baseline burst amplitude and expressed as a function of the burst number to eliminate the locomotor period interindividual variability. Burst number -1 corresponds to the final complete burst before the CS HFS, and burst number 1 corresponds to the first complete burst recorded after the CS HFS application. As shown in Figures 10D1,E, the CS HFS was inefficient in modifying the motor burst amplitude recorded from the rL2 ventral root, but it induced a STP of the locomotor-related bursts recorded from both the rL5 and the 


\section{A1}

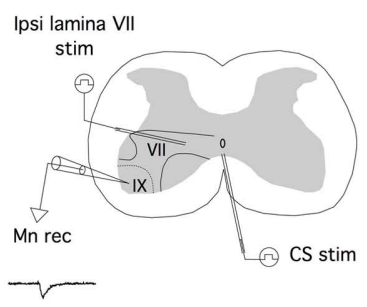

B1

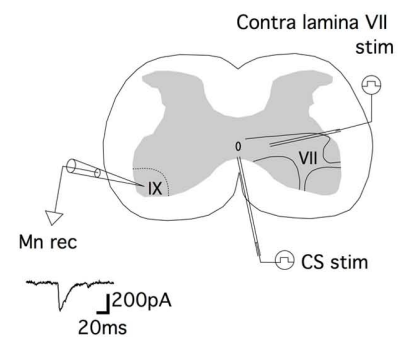

\section{A2}

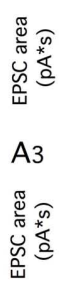

B2

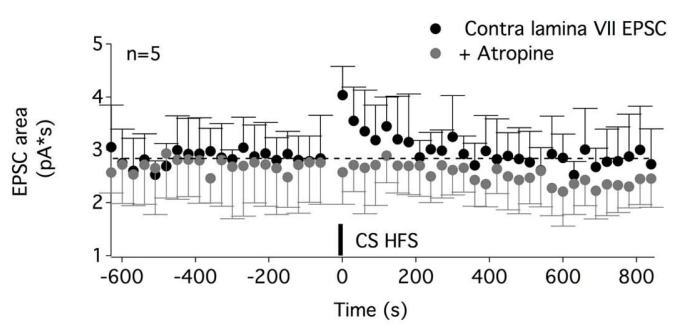

FIGURE 8 | The muscarinic commissural transmission induced an STP of the contralateral lamina VII glutamatergic inputs but not of the ipsilateral lamina VII glutamatergic inputs. (A) Schema of the experimental procedure (A1). A tungsten electrode was placed on the ventral commissure (CS stim) and another in the ipsilateral lamina VII (ipsi lamina VII stim) to record ipsi lamina VII EPSCs from motoneurons (Mnrec) held at $-60 \mathrm{mV}$, as exemplified by the trace in (A1). (A2) Plot of the mean ipsi lamina VII EPSC area as a function of time before and after CS HFS. No STP was induced in these experimental conditions.
(A3) Plot of the mean com-AMPA EPSC area as a function of time before and after a CS HFS in five out of the eight neurons tested in the (A2) condition, showing the induction of com-AMPA EPSC STP in these neurons. (B). Schema of the experimental procedure (B1). Contralateral lamina VII EPSCs (contra lamina VII) were evoked in motoneurons as exemplified by the trace in (B1). (B2) The time course of the mean contra lamina VII EPSC area as a function of time before and after a CS HFS in the absence (black circles) or the presence of atropine (200 $\mu \mathrm{M}$, gray circles).
IL5 ventral roots (burst-STP). CS HFS-induced potentiation of burst amplitude dissipated in about $30 \mathrm{~s}$ after induction in all of the seven spinal cords tested. The subsequent supplementation of the aCSF with atropine completely occluded burst-STP expression (Figures 10D2,E) and, in contrast, led to a transient pause in locomotor activity immediately following the CS HFS (Figure 10D2). The effects of atropine were reversed after a 30-45-min washout (data not shown). The burst-STP appears specific to the CS stim, as the same stimulation paradigm applied to the L5 dorsal root failed to induce changes in the motor burst amplitude (Figure 10F). These data suggest that during fictive locomotion, the activation of the muscarinic commissural transmission can transiently amplify the bursting activity of lumbar motoneurons.

\section{DISCUSSION}

The present work, to our knowledge, represents the first detailed description of the electrophysiological properties of the synapses between cholinergic partition cells and motoneurons. In addition, our study reveals that the specific synaptic activation of commissural cholinergic inputs mediate pathway-specific short-lasting synaptic potentiation in ventral spinal motor networks.

\section{MUSCARINIC RECEPTORS IN THE SPINAL MOTOR NETWORKS}

In the present study, we showed that a commissural cholinergic transmission sustained by muscarinic receptor activation could be recorded from lumbar motoneurons. These data further emphasize the salient role of muscarinic receptors in motor neural assemblies. In addition to the nicotinic-mediated effects of acetylcholine (Ach), muscarinic-mediated ones have been, indeed, reported at all neural levels involved in locomotion: in supraspinal structures (Garcia-Rill and Skinner, 1987; Le Ray et al., 2004; Smetana et al., 2007, 2010) and at the spinal cord level (Smith et al., 1988; Kurihara et al., 1993; Cowley and Schmidt, 1994; Alaburda et al., 2002; Miles et al., 2007; Anglister et al., 2008).

The pharmacological experiments performed in the present study show the following: (1) the CM inputs cause the closure of the M-current; (2) the CM transmission involved at least the M2, $\mathrm{M} 3$, and M4 receptors.

In the literature, the M-current is classically associated with the M1/M3 muscarinic receptors (for review see Brown, 2010). The lack of effect of pirenzepine on the muscEPSP amplitude, however, suggests that M1 receptor activation is not involved in the CM transmission. Lumbar motoneurons should nevertheless express M1 muscarinic receptors because pirenzepine significantly inhibits the oxotremorine-induced depolarization observed in synaptically isolated motoneurons. The role of the M3 receptors at commissural cholinergic terminals appears to be more complex. The muscEPSP amplitude and duration enhancement observed in the presence of 4-DAMP suggests that the M3 receptor activation counteracts the Ach effects. 4-DAMP, however, inhibited the oxotremorine-induced depolarization, indicating that a postsynaptic M3 receptor activation depolarises the motoneurons. Altogether, these data suggest that the $\mathrm{M} 3$ receptors are inhibitory autoreceptors in commissural cholinergic terminals. These results 

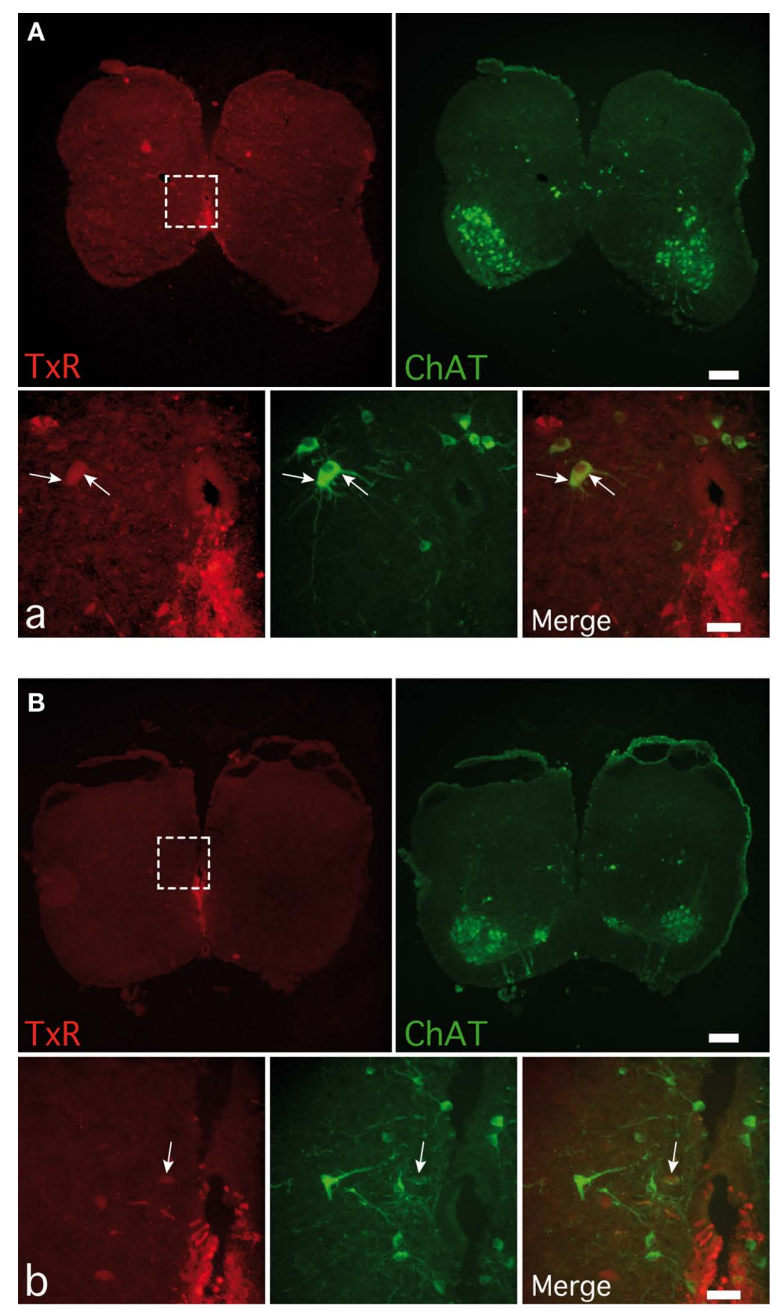

C

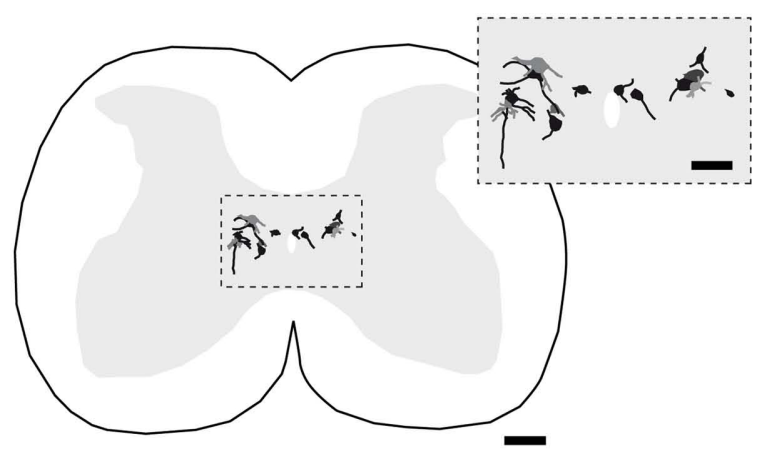

FIGURE 9 | Detection of cholinergic commissural interneurons in spinal cord slices. Photomicrographs of commissural interneurons (CINs) retrogradely labeled with Texas red (TxR), applied ventrally to the central canal and immunopositive for CholineAcetylTransferase (ChAT) in lamina VII (A) and close to the central canal (B). The areas boxed in $(\mathbf{A}, \mathbf{B})$ are shown at a higher magnification in $(a, b)$ photomicrographs. Arrows point to the double-labeled CINs. (C) A normalized spinal cord transverse section illustrating the location of all the double-labeled CINs observed. The area boxed is shown at a higher magnification in the inset. [Calibration bars: (A-C), $200 \mu \mathrm{m}$; (a,b), $50 \mu \mathrm{m}$; inset, $100 \mu \mathrm{m}$. are in agreement with numerous studies showing that Ach release is subject to strong auto-inhibition via the activation of presynaptic muscarinic receptors (for review see Brown, 2010). In the hippocampus, activation of $\mathrm{M} 1$ receptors has been shown to increase the glutamatergic release trough the inhibition of small conductance $\mathrm{Ca}^{2+}$ activated $\mathrm{K}^{+}$channels (SK) present in dendritic spines (Giessel and Sabatini, 2010). In the same way, delayed rectifier $\mathrm{K}^{+}$ channels have been shown to influence neurotransmitter release in numerous synapses (see for example: Shimada et al., 2007; Martel et al., 2011). Such regulations of Ach release in commissural terminals could explain the increase in muscEPSP amplitude reported in the presence of the SK (apamin) or delayed rectifier (4-AP) channels blockers.

\section{PARTITION CELLS AND LAMINA X NEURONS ARE THE SOURCE OF THE COMMISSURAL MUSCARINIC TRANSMISSION}

Premotor IN populations monosynaptically projecting to lumbar motoneurons have recently been described (Stepien et al., 2010). The analysis of the connectivity between cholinergic neurons and motoneurons reveals the existence of both ipsilaterally and bilaterally projecting neurons. In our study, high divalent cationcontaining aCSF was used through out the electrophysiological experiments to depress the polysynaptic transmission (Cazalets et al., 1995). Moreover, we show that the CM transmission persists in the presence of blockers of fast excitatory and inhibitory synaptic transmission (in the presence of DNQX, strychnine, and gabazine). We could then claim that the CM transmission we studied is essentially monosynaptic. The possibility that CS electrical stimulations could have activated ipsilateral propriospinal pathways cannot be completely excluded. However, the small size of the stimulating electrodes as well as the stimulation parameters used (see Materials and Methods) should have confined the stimulating currents to the ventral commissure thereby limiting the stimulation of non-commissural fibers.

Since the pioneer studies describing spinal cholinergic neurons, clear distinctions have been made between central canal cells of lamina X and partition cells located in lamina VII (Barber et al., 1984; Phelps et al., 1984). ChAT immunoreactivity in the spinal cord has indeed revealed cells of small size with oval or rounded cell bodies located in lamina X (central canal cells) and larger, multipolar cells in the midportion of the intermediate gray matter (partition cells). In addition to these topological and morphological differences, Michael et al. (1997) have shown that TrkA immunoreactivity is present in a high percentage of partition cells but only a small proportion of central canal cells suggesting that these two groups of cholinergic neurons constituted different interneuronal populations. According to this cellular classification and to the extent of lamina X (Watson et al., 2008), we show in the present study that muscEPSPs largely originated from cholinergic partition cells located in lamina VII and lamina X central canal cells retrogradely labeled from the ventral commissure.

The recently described subpopulation of cholinergic V0 INs, Pixt2 neurons preferentially connect ipsilateral lumbar motoneurons (Zagoraiou et al., 2009). The cholinergic retrogradely labeled neurons observed in the present study could then be part of the bilaterally projecting cholinergic partition cells identified by Stepien et al. (2010) whose description closely corresponds to the 
A

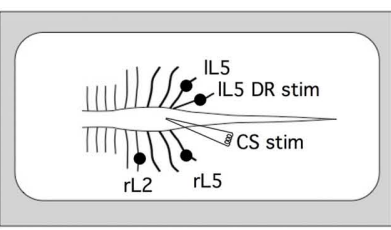

B1 NMA-5HT

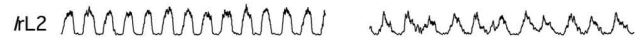

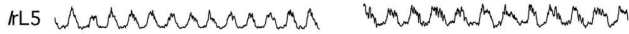

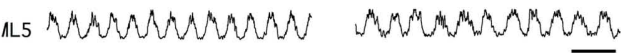
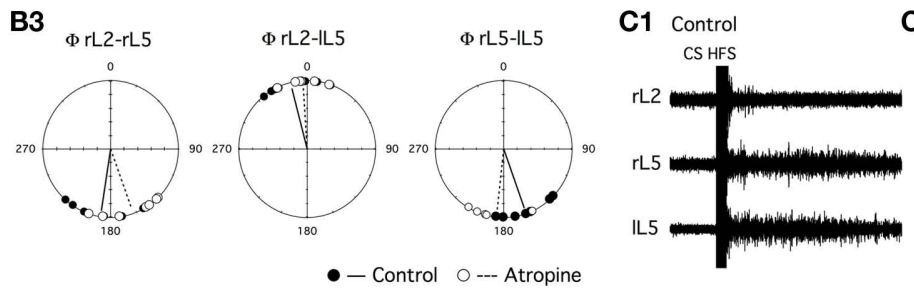

C2 + Atropine

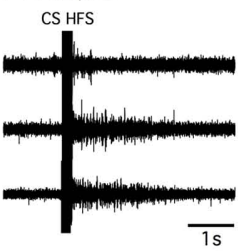

D2 + Atropine

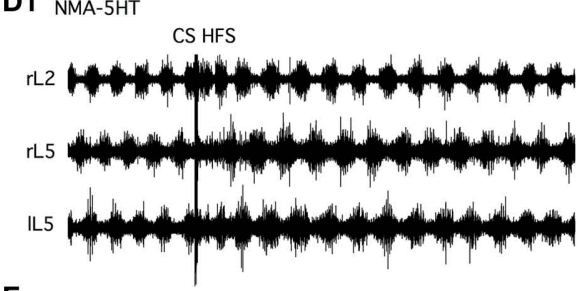

E
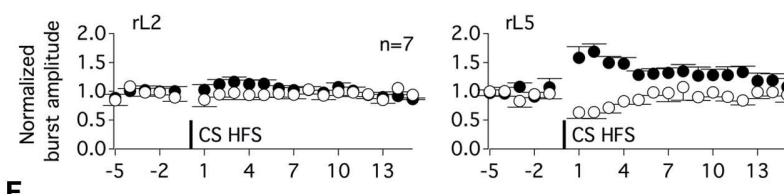

F
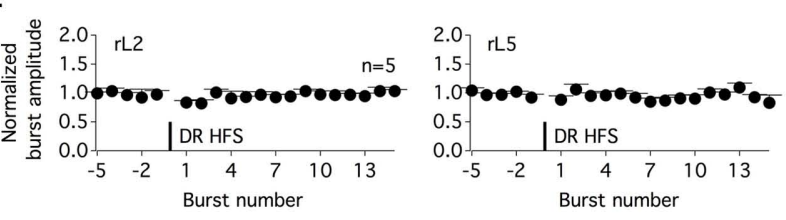

CS HFS
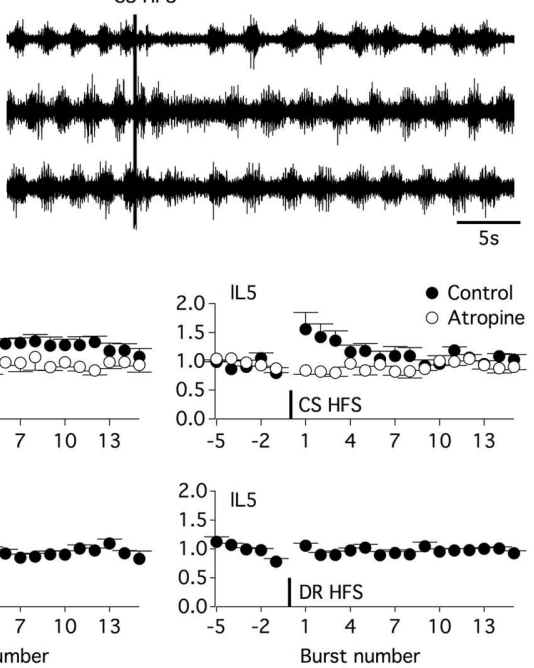

FIGURE 10 | Commissural muscarinic transmission transiently amplifies the motor output during fictive locomotion. (A) Schematic diagram of the experimental procedure. Fictive locomotion was recorded from the right lumbar 2, 5 and left lumbar 5 ventral roots (rL2, rL5, IL5) in the isolated spinal cord preparation. A tungsten bipolar electrode was used to stimulate the ventral commissure (CS stim). (B) Integrated recordings (/) of fictive locomotion induced by the bath application of NMA and $5 \mathrm{HT}(16 \mu \mathrm{M}$ each) in the absence (B1) or presence of atropine [200 $\mu$ M (B2)]. Circular analysis of the motor burst activity relationships $(\Phi)$ in the control condition (black circles and trace) and in an atropine-containing aCSF (white circles and dashed trace) (B3). (C) CS HFS $(100 \mathrm{~Hz}, 20$ shocks) induced transient activity in a quiescent spinal cord preparation in the control condition (C1) and in the presence of atropine (C2). (D). CS HFS application during NMA/5HT-induced fictive locomotion in the absence (D1) or presence of atropine (D2). (E) Summary plots of the normalized burst amplitude as a function of the burst number for the three-recorded ventral roots. Burst number -1 corresponds to the final complete burst recorded before the CS HFS, and burst number 1 corresponds to the first complete burst observed after the CS HFS. In the control conditions (black circles), CS HFS-induced a short-term potentiation of the amplitude of motor bursts recorded from the two L5 ventral roots that was occluded in the presence of atropine (white circles). (F) Summary plots of the fictive locomotion normalized burst amplitude as a function of the burst number for the three-recorded ventral roots before and after the application of a $100-\mathrm{Hz}, 20$ shock stim to the IL5 dorsal root (DR HFS).
TxR retrogradely labeled cells observed in our report. Among the different types of cholinergic synapses connecting motoneurons, large terminals, which are termed C-boutons, located on motoneuronal somata and proximal dendrites, have been described. Cholinergic V0INs have been shown to be the unique source of the C-boutons (Miles et al., 2007; Zagoraiou et al., 2009). The pharmacological analysis performed in our study indicates that the functional organization of the commissural cholinergic synapses differs from the one reported for the C-terminals. We indeed show that the CM transmission is coupled to the M-current and, consequently, to the M1/M3/M5 family (Delmas and Brown, 2005). C-boutons, in contrast, are exclusively associated with the M2 muscarinic receptors and are in close apposition with $\mathrm{K}_{\mathrm{v}} 2.1$ and $\mathrm{K}_{\mathrm{Ca}}$ currents (Miles et al., 2007; Zagoraiou et al., 2009). In the study of Zagoraiou et al. (2009), the authors observed the disappearance of ChAT immunopositive axosomatic C-boutons in motoneurons following the genetic inactivation of V0c INs but analyses to characterize potential changes in cholinergic synapses at the dendritic 
tree level were not carried out. M-channels are mainly expressed at the dendritic level in neurons such as pyramidal cells in the hippocampus (see for example Lawrence et al., 2006). We can then hypothesize that part of the cholinergic INs, as the commissural ones, could connect lumbar motoneurons through axodendritic synapses associated with M-channels. Cholinergic spinal INs could then be divided into, at least, two different populations based on their synapse type on motoneurons: the Pitx2 neurons that project bilaterally on motoneurons through C-boutons and other lamina X neurons and some partition cells that project contralaterally through cholinergic synapses associated with the M-channel and presumably with the M1-M3 receptor family. It has also to be noted that this study has been performed in rat spinal cord slices. Discrepancies between our data and data from earlier studies could be due to differences in rat and mouse cholinergic system.

\section{SHORT-TERM PLASTICITY OF COMMISSURAL GLUTAMATERGIC INPUTS}

We have found that the activation of the commissural cholinergic system triggers a pathway-specific STP of commissural glutamatergic inputs in the spinal cord. Moreover, activation of the commissural transmission during fictive locomotion causes a significant muscarinic-dependent increase in motor output. Interactions between the cholinergic and glutamatergic neurotransmitter systems have been shown to influence various forms of synaptic plasticity within different nervous structures (see for example: Martella et al., 2009; Drever et al., 2011). In these models, muscarinic receptor activation has been shown not only to modulate synaptic plasticity induced by HFS, but also to induce long-term plasticity itself. Our study shows that such synaptic amplification mechanisms are present in the spinal cord networks. The lack of effect of the M3-preferring antagonist 4-DAMP on both the baseline com-AMPA-EPSC area and the STP indicate that these receptors are not involved in the modulation of glutamatergic transmission and in the musc-STP. In contrast, the M-current blocker XE991 decreased the baseline com-AMPA-EPSC area without altering the musc-STP. These results could be explained by a presynaptic control of the glutamatergic transmission by the M-current, as shown, for example, in hippocampal synaptosomes (Martire et al., 2004).

In the literature, as in the present study, the electrical stimulations used to activate muscarinic pathways in the central nervous system regularly induces glutamatergic-mediated events preceding long-lasting muscarinic responses (Brown, 2010). These observations lead to the idea that glutamate and Ach could be co-released from the same synaptic terminals. This is the case in, for example, cultured basal forebrain neurons, where Ach can inhibit its own release and also that of glutamate (Allen et al., 2006). Modulations of one neurotransmitter system then impact the other. In the present study, however, a bath application of atropine or an inhibition of the $\mathrm{M} 3$ receptors did not modify the baseline comAMPA-EPSC amplitude. These data tend to suggest that different synaptic terminals release Ach and commissural glutamate onto motoneurons, although a possible co-release cannot be completely ruled out.

An interesting characteristic of the musc-STP described in the present paper is that it appears specific of the contralateral lamina
VII glutamatergic inputs. Indeed, CS HFS potentiated com-lamina VII EPSCs but failed to modify the ipsi lamina VII EPSC area. The question arises as to whether this pathway specificity could be artifactual and linked to the direct electrical stimulation of commissural glutamatergic axons by the CS stimulating electrode. In our opinion, the occlusion of com-lamina VII EPSC STP by atropine excludes this possibility. These data indeed indicate that the amplification of com-lamina VII EPSC area relies on Ach release and muscarinic receptor activation and not on an electrically induced increase in glutamatergic axon excitability.

In the last part of this study, using the in vitro spinal cord preparation, we showed that commissural cholinergic pathways could modulate motoneuron output during fictive locomotion. As the T13-L2 segments have been described as the main arrhythmogenic area in the rodent spinal cord (Cazalets, 2000; Kiehn, 2006), the effects of CS HFS were tested at the L5 level to minimize the effect of the CS HFS on the locomotor CPG. While musc-STP was observed in every lumbar spinal cord slice, the L5 CS HFS effects were restricted to the L5 segments in the whole spinal cord preparation. The application of serotonin, used to induce fictive locomotion, could partly explain this lack of propagation as this neuromodulator has been shown in different studies to inhibit part of the synaptic transmission along the spinal cord (see for example: Schmidt and Jordan, 2000; Barriere et al., 2008). In the same series of experiments, we observed that L5 CS HFS triggered a transient pause in fictive locomotion in the presence of atropine. In spinal cord slices, commissural stimulations were applied in the presence of blockers of the fast inhibitory transmission to isolate glutamatergic transmission. In the whole preparation in contrast, the spinal cord was superfused with NMA-5HT containing aCSF. In these experimental conditions, the stimulation of inhibitory commissural interneurons could then partly account for the gap in motor bursting observed after L5 CS HFS in the presence of atropine.

\section{PHYSIOLOGICAL RELEVANCE}

The present results are of particular interest with regard to the recent data obtained in mice in which all of the cholinergic V0 INs were genetically inactivated (V0 $c^{\text {off }}$ mouse; Zagoraiou et al., 2009). Using EMG recordings, these authors showed that the locomotor parameters of ${\mathrm{V} 0 \mathrm{c}^{\text {off }}}$ mice were unaltered compared to control animals. The lack of atropine effects that we reported on phase coupling during fictive locomotion further supports the fact that cholinergic INs are not directly involved in motor pattern generation. In contrast, $\mathrm{V} 0 \mathrm{c}$ INs seem to play a major role in the modulation of the motor output. V0 $\mathrm{c}^{\text {off }}$ mice indeed present a significant impairment in gastrocnemius burst amplitude modulation during swimming (Zagoraiou et al., 2009). The authors hypothesize that this effect could be explained by a failure to activate $\mathrm{M} 2$ muscarinic receptors at $\mathrm{C}$-boutons on motoneurons. Our study provides an additional explanation. Cholinergic transmission could indeed be altered in $\mathrm{VO} \mathrm{c}^{\text {off }}$ mice, leading to the occlusion of musc-STP and then to the impossibility for the locomotor CPG to increase glutamatergic inputs on motor neuron pools during swimming.

We have, so far, no idea as to how commissural lamina $\mathrm{X}$ neurons as well as partition cells participate in locomotion 
(see Huang et al., 2000). These neurons could also play a major role in postural control, which likely require synchronized activation.

Bilaterally projecting cholinergic partition cells have been shown to present a high degree of synaptic specificity with a preferential targeting of corresponding motor pools (Stepien et al., 2010). If we hypothesize that bilaterally projecting partition cells originate CM transmission and then musc-STP, a very interesting question that arises is whether commissural glutamatergic transmission is potentiated in functionally identical motoneuron pools in the two different sides of the spinal cord.

\section{REFERENCES}

Alaburda, A., Perrier, J. F., and Hounsgaard, J. (2002). An M-like outward current regulates the excitability of spinal motoneurones in the adult turtle. J. Physiol. 540, 875-881.

Allen, T. G., Abogadie, F. C., and Brown, D. A. (2006). Simultaneous release of glutamate and acetylcholine from single magnocellular "cholinergic" basal forebrain neurons. J. Neurosci. 26, 1588-1595.

Anglister, L., Etlin, A., Finkel, E., Durrant, A. R., and Lev-Tov, A. (2008). Cholinesterases in development and disease. Chem. Biol. Interact. 175, 92-100.

Barber, R. P., Phelps, P. E., Houser, C. R., Crawford, G. D., Salvaterra, P. M., and Vaughn, J. E. (1984). The morphology and distribution of neurons containing choline acetyltransferase in the adult rat spinal cord: an immunocytochemical study. $J$. Comp. Neurol. 229, 329-346.

Barriere, G., Tartas, M., Cazalets, J. R., and Bertrand, S. S. (2008). Interplay between neuromodulatorinduced switching of short-term plasticity at sensorimotor synapses in the neonatal rat spinal cord. $J$. Physiol. 586, 1903-1920.

Brown, D. A. (2010). Muscarinic acetylcholine receptors (mAChRs) in the nervous system: some functions and mechanisms. J. Mol. Neurosci. 41, 340-346.

Brown, D. A., and Passmore, G. M. (2009). Neural KCNQ (Kv7) channels. Br. J. Pharmacol. 156, 1185-1195.

Cazalets, J. R. (2000). "Organization of the spinal locomotor network in neonatal rat," in Neurobiology of Spinal Cord Injury, ed. R. Kalb and S. M. Stritmatter (Totowa, NJ: Humana Press Inc.), 89-111.

Cazalets, J. R., Borde, M., and Clarac, F. (1995). Localization and organization of the central pattern generator for hindlimb locomotion in newborn rat. J. Neurosci. 15, 4943-4951.

Cowley, K. C., and Schmidt, B. J. (1994). A comparison of motor patterns induced by $\mathrm{N}$-methyl-D-aspartate, acetylcholine and serotonin in the in vitro neonatal rat spinal cord. Neurosci. Lett. 171, 147-150.

Delmas, P., and Brown, D. A. (2005). Pathways modulating neural $\mathrm{KCNQ} / \mathrm{M} \quad(\mathrm{Kv} 7)$ potassium channels. Nat. Rev. Neurosci. 6, 850-862.

Drever, B. D., Riedel, G., and Platt, B. (2011). The cholinergic system and hippocampal plasticity. Behav. Brain Res. 221, 505-514.

Enjin, A., Rabe, N., Nakanishi, S. T., Vallstedt, A., Gezelius, H., Memic, F, Lind, M., Hjalt, T., Tourtellotte, W. G., Bruder, C., Eichele, G., Whelan, P. J., and Kullander, K. (2010). Identification of novel spinal cholinergic genetic subtypes disclose Chodl and Pitx2 as markers for fast motor neurons and partition cells. J. Comp. Neurol. 518, 2284-2304.

Fernandez de Sevilla, D., Nunez, A., Borde, M., Malinow, R., and Buno, W. (2008). Cholinergic-mediated IP3-receptor activation induces long-lasting synaptic enhancement in CA1 pyramidal neurons. $J$. Neurosci. 28, 1469-1478.

Garcia-Campmany, L., Stam, F. J., and Goulding, M. (2010). From circuits to behavior: motor networks in vertebrates. Curr. Opin. Neurobiol. 20, 116-125.

Garcia-Rill, E., and Skinner, R. D. (1987). The mesencephalic locomotor region. I. Activation of a medullary projection site. Brain Res. $411,1-12$.

Giessel, A. J., and Sabatini, B. L. (2010). M1 muscarinic receptors boost synaptic potentials and calcium influx in dendritic spines by inhibiting postsynaptic SK channels. Neuron 68, 936-947.

Grillner, S., Wallen, P., Saitoh, K., Kozlov, A., and Robertson, B. (2008). Neural bases of goal-directed locomotion in vertebrates - an overview. Brain Res. Rev. 57, 2-12.

Huang, A., Noga, B. R., Carr, P. A., Fedirchuk, B., and Jordan, L. M. (2000). Spinal cholinergic neurons activated during locomotion: localization and electrophysiological

Our study opens the door to the exciting possibility that the cholinergic system, by changing synaptic coupling inside the locomotor CPG, could amplify specific synaptic inputs in a taskrelated manner. Partition cells and lamina $\mathrm{X}$ neurons, by their "strategic position" at the interface between sensory, autonomic and ventral systems, appear particularly suitable to exert such neuromodulatory control.

\section{ACKNOWLEDGMENTS}

The authors are grateful to Laura Cardoit for technical assistance and to Dr. Frédéric Nagy for critical reading of the manuscript.

characterization. J. Neurophysiol. 83, $3537-3547$.

Kiehn, O. (2006). Locomotor circuits in the Mammalian spinal cord. Annu. Rev. Neurosci. 29, 279-306.

Kiehn, O. (2011). Development and functional organization of spinal locomotor circuits. Curr. Opin. Neurobiol. 21, 100-109.

Kiehn, O., and Kjaerulff, O. (1996). Spatiotemporal characteristics of 5HT and dopamine-induced rhythmic hindlimb activity in the in vitro neonatal rat. J. Neurophysiol. 75 1472-1482.

Kurihara, T., Suzuki, H., Yanagisawa, M., and Yoshioka, K. (1993). Muscarinic excitatory and inhibitory mechanisms involved in afferent fibreevoked depolarization of motoneurones in the neonatal rat spinal cord. Br. J. Pharmacol. 110, 61-70.

Lawrence, J. J., Saraga, F., Churchill, J. F., Statland, J. M., Travis, K. E., Skinner, F. K., and Mcbain, C. J. (2006). Somatodendritic Kv7/KCNQ/M channels control interspike interval in hippocampal interneurons. $J$. Neurosci. 26, 12325-12338.

Le Ray, D., Brocard, F., and Dubuc, R. (2004). Muscarinic modulation of the trigemino-reticular pathway in lampreys. J. Neurophysiol. 92, 926-938.

Martel, P., Leo, D., Fulton, S., Berard, M., and Trudeau, L. E. (2011). Role of Kvl potassium channels in regulating dopamine release and presynaptic D2 receptor function. PLoS ONE 6, e20402. doi:10.1371/journal.pone. 0020402

Martella, G., Tassone, A., Sciamanna, G., Platania, P., Cuomo, D., Viscomi, M. T., Bonsi, P., Cacci, E., Biagioni, S., Usiello, A., Bernardi, G., Sharma, N., Standaert, D. G., and Pisani, A. (2009). Impairment of bidirectional synaptic plasticity in the striatum of a mouse model of DYT1 dystonia: role of endogenous acetylcholine. Brain 132, 2336-2349.

Martire, M., Castaldo, P., D’amico, M., Preziosi, P., Annunziato, L., and Taglialatela, M. (2004). M channels containing KCNQ2 subunits modulate norepinephrine, aspartate, and GABA release from hippocampal nerve terminals. J. Neurosci. 24, 592-597.

McCrea, D. A., and Rybak, I. A. (2008). Organization of mammalian locomotor rhythm and pattern generation. Brain Res. Rev. 57, 134-146.

Michael, G. J., Kaya, E., Averill, S., Rattray, M., Clary, D. O., and Priestley, J. V. (1997). TrkA immunoreactive neurones in the rat spinal cord. $J$. Comp. Neurol. 385, 441-455.

Miles, G. B., Hartley, R., Todd, A. J., and Brownstone, R. M. (2007) Spinal cholinergic interneurons regulate the excitability of motoneurons during locomotion. Proc. Natl. Acad. Sci. U.S.A. 104, 2448-2453.

O'Donovan, M. J., Bonnot, A., Mentis, G. Z., Arai, Y., Chub, N., Shneider, N. A., and Wenner, P. (2008). Imaging the spatiotemporal organization of neural activity in the developing spinal cord. Dev. Neurobiol. 68, 788-803.

Phelps, P. E., Barber, R. P., Houser, C. R., Crawford, G. D., Salvaterra, P. M., and Vaughn, J. E. (1984). Postnatal development of neurons containing choline acetyltransferase in rat spinal cord: an immunocytochemical study. J. Comp. Neurol. 229, 347-361.

Roberts, A., Li, W. C., Soffe, S. R., and Wolf, E. (2008). Origin of excitatory drive to a spinal locomotor network. Brain Res. Rev. 57, 22-28.

Schmidt, B. J., and Jordan, L. M. (2000). The role of serotonin in reflex modulation and locomotor rhythm production in the mammalian spinal cord. Brain Res. Bull. 53, 689-710.

Shimada, H., Uta, D., Nabekura, J., and Yoshimura, M. (2007). Involvement of $\mathrm{Kv}$ channel subtypes on GABA release in mechanically dissociated neurons from the rat substantia nigra. Brain Res. 1141, 74-83.

Smetana, R., Juvin, L., Dubuc, R., and Alford, S. (2010). A parallel cholinergic brainstem pathway for enhancing locomotor drive. Nat. Neurosci. 13, 731-738. 
Smetana, R. W., Alford, S., and Dubuc, R. (2007). Muscarinic receptor activation elicits sustained, recurring depolarizations in reticulospinal neurons. J. Neurophysiol. 97, 3181-3192.

Smith, J. C., Feldman, J. L., and Schmidt, B. J. (1988). Neural mechanisms generating locomotion studied in mammalian brain stem-spinal cord in vitro. FASEB J. 2, 2283-2288.

Stepien, A. E., Tripodi, M., and Arber, S. (2010). Monosynaptic rabies virus reveals premotor network organization and synaptic specificity of cholinergic partition cells. Neuron 68, 456-472.

Watson, C., Paxinos, G., and Kayalioglu, G. (2008). The Spinal Cord. Amsterdam: Elsevier.

Zagoraiou, L., Akay, T., Martin, J. F., Brownstone, R. M., Jessell, T. M., and Miles, G. B. (2009). A cluster of cholinergic premotor interneurons modulates mouse locomotor activity. Neuron 64, 645-662.

Zhang, H. M., Chen, S. R., Cai, Y. Q., Richardson, T. E., Driver, L. C., Lopez-Berestein, G., and Pan, H. L. (2009). Signaling mechanisms mediating muscarinic enhancement of GABAergic synaptic transmission in the spinal cord. Neuroscience 158, 1577-1588.

Conflict of Interest Statement: The authors declare that the research was conducted in the absence of any commercial or financial relationships that could be construed as a potential conflict of interest.

Received: 30 August 2011; accepted: 13 October 2011; published online: 04 November 2011.
Citation: Bertrand SS and Cazalets J$R$ (2011) Cholinergic partition cells and lamina $X$ neurons induce a muscarinicdependent short-term potentiation of commissural glutamatergic inputs in lumbar motoneurons. Front. Neural Circuits 5:15. doi: 10.3389/fncir.2011.00015 Copyright (C) 2011 Bertrand and Cazalets. This is an open-access article subject to a non-exclusive license between the authors and Frontiers Media SA, which permits use, distribution and reproduction in other forums, provided the original authors and source are credited and other Frontiers conditions are complied with. 